\title{
Lorsque le « chez-soi » se dérobe
}

Invisibilisation des travailleurs saisonniers en montagne, le cas des "nourris-logés »

When "at home" slips away. Invisibilisation of the seasonal workers in mountain: the case of "board and lodging"

\section{Liza Baghioni}

\section{(2) OpenEdition}

\section{Journals}

Édition électronique

URL : https://journals.openedition.org/tc/6408

DOI : $10.4000 /$ tc. 6408

ISBN : $1952-420 X$

ISSN : 1952-420X

Éditeur

Éditions de l'EHESS

\section{Édition imprimée}

Date de publication : 15 juin 2012

Pagination : 230-249

ISBN : 2-7351-1512-7

ISSN : 0248-6016

\section{Référence électronique}

Liza Baghioni, «Lorsque le «chez-soi » se dérobe », Techniques \& Culture [En ligne], 58 | 2012, mis en ligne le 30 octobre 2012, consulté le 29 septembre 2022. URL : http://journals.openedition.org/tc/ 6408 ; DOI : https://doi.org/10.4000/tc.6408 


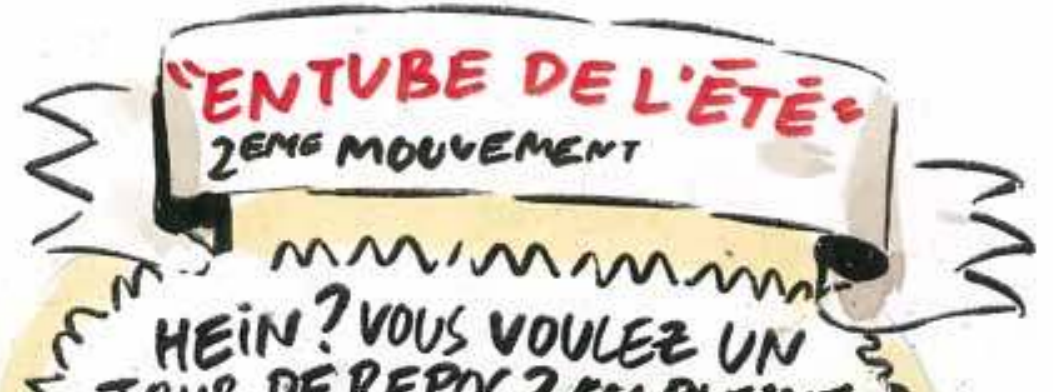
S JOUR DEREPOS? EN PLEINE? \$PERIODEDE VACANCES? LELIQUIDE-VAISSELLEVOUS?

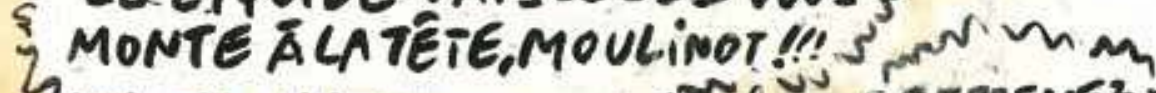
hung MNun Nan

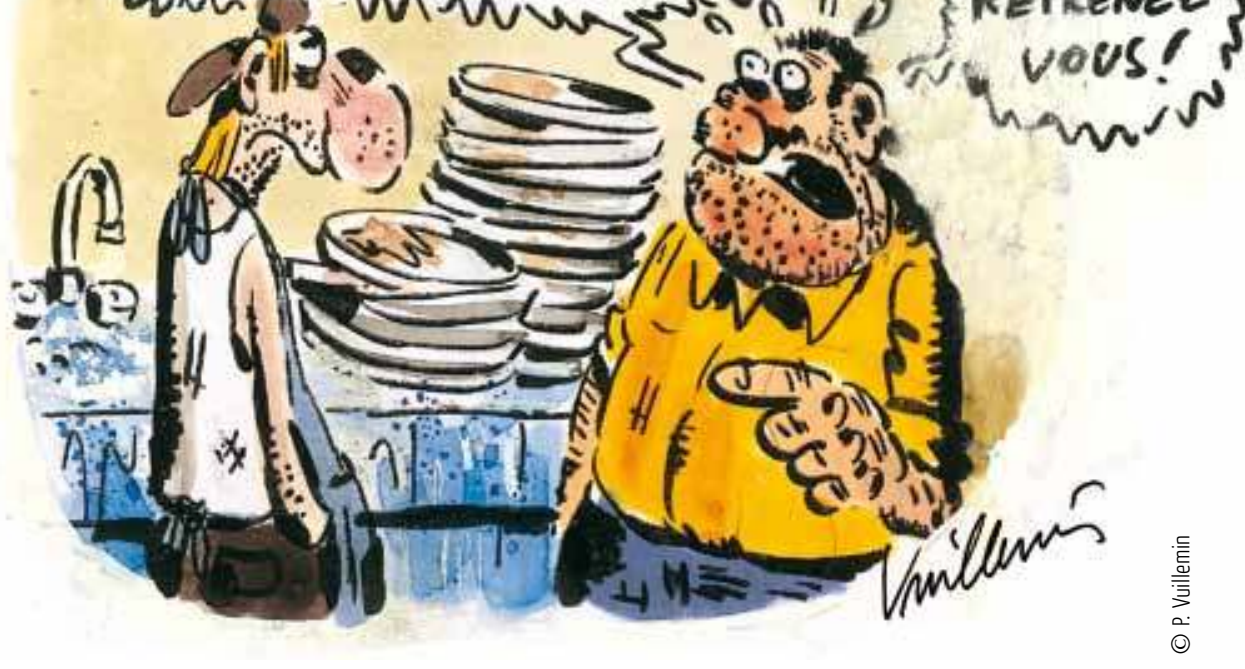




\section{Liza Baghioni}

Doctorante à I'IDEMEC - UMR 7307

liza_baghioni@hotmail.com

Techniques E Culture 58, 2012/1: 230-249

\section{LORSQUE LE « CHEZ-SOl » SE DÉROBE}

\section{Invisibilisation des travailleurs saisonniers en montagne: le cas des "nourris-logés»}

L'illustration satirique de Vuillemin nous permet de pointer d'entrée de jeu une certaine disjonction entre tourisme et travail, par ailleurs relayée dans les débats déjà anciens qui opposent ces deux versants des rythmes sociaux (Veblen 1970 [1899] ; Friedmann 1956; Dumazedier 1962). L'analyse du travail constitue ainsi une sorte d'angle mort des recherches académiques sur le tourisme et les lieux de vacances. Une revue de la littérature scientifique permet d'appuyer ce constat. Si les enquêtes macro-sociologiques et statistiques qui lient tourisme et migrations de travail sont nombreuses (Viard 2002; Dehoorne 2002), il existe en revanche peu de travaux ethnographiques qui questionnent les effets de cette forme d'emploi sur les salariés. Du côté anglo-saxon, les rares études qualitatives sur les « migrants tourist-workers » en témoignent. Certains auteurs (Bianchi 2000 ; Uriely 2001) expliquent que cette absence de travaux est en partie liée à la rigidité des découpages disciplinaires (anthropologie du tourisme, sociologie du travail, anthropologie économique), ainsi qu'à une hiérarchisation des sujets de recherche. Comme le rappelle Jean Michaud, l'anthropologie du tourisme a eu tendance à se constituer comme un objet autonome, portant principalement son regard sur la « figure du touriste » ou encore sur le tourisme globalisé « comme solution, comme catalyseur d'acculturation et comme forme perverse de développement » (Michaud 2001 : 15).

En France, la majorité des travaux à propos des saisonniers du tourisme (on en dénombre $420000^{1}$ sur le territoire français) sont issus de la commande sociale (régions, communes, syndicats, etc.) ${ }^{2}$. Pourtant, cette main d'œuvre - caractérisée par une soumission aux variations saisonnières, affiliée à des régimes d'emploi divers, contrainte à une certaine mobilité ou pluriactivité, travaillant à contre-rythme des
Habiter à part, hors des lieux collectifs, c'est disposer d'un lieu protégé d'où la pression du corps social sur le corps individuel est écartée, où le pluriel des stimuli est filtré ou, idéalement, devrait l'être.

(De Certeau 1994 : 207) 
temps sociaux dominants - cristallise de nombreux débats autour du sens et de la place du travail dans nos sociétés (Kergoat \& al. 1998).

Quelques sociologues se sont toutefois intéressés à la spécificité des activités de travail dans les lieux de vacances. Ils décrivent l'obligation pour les salariés d'être en constante « représentation de soi » (Réau 2006 : 76) et relèvent une forme de « dénégation organisée de la relation marchande » (Giraud 2007 : 16). Ces travaux renvoient à des processus d'invisibilisation, ici structurés par l'organisation du travail. Il en résulte que l'analyse des conditions de travail des personnes qui accueillent les touristes permet d'éclairer certains mécanismes sociaux à l'œuvre dans ces temps spécifiques que sont les vacances. Ainsi serait-il possible d'envisager « l'enchantement touristique », maintes fois mobilisé par les anthropologues du tourisme, comme une coproduction entre travailleurs et vacanciers. C'est le point de vue que développent Godfrey Baldacchino (1997) et George Gmelch (2003) dans une analyse des « host-guest intercations » centrée sur l'expérience des travailleurs dans des îles caribéennes et méditerranéennes. Ces recherches permettent de constater que les employés (bien que très critiques vis-à-vis des touristes) ont tendance à intérioriser la vision enchantée du travail destinée aux vacanciers. La façon dont ils pensent et construisent leur place au sein du système touristique est ainsi largement déterminée par des représentations liées aux territoires sur lesquels ils restent, passent ou s'intègrent (ils sont attirés par des lieux de travail « paradisiaques » ou revendiquent leur volonté de « vivre et travailler au pays », etc.). Cette piste permettrait de transcender la question redondante (posée par le monde politico-institutionnel) d'une saisonnalité subie ou choisie.

La voie est ouverte et le chemin reste hypothétique. Si, au cœur des lieux touristiques, l'invisibilisation du travail et des travailleurs est un ressort de l'enchantement des

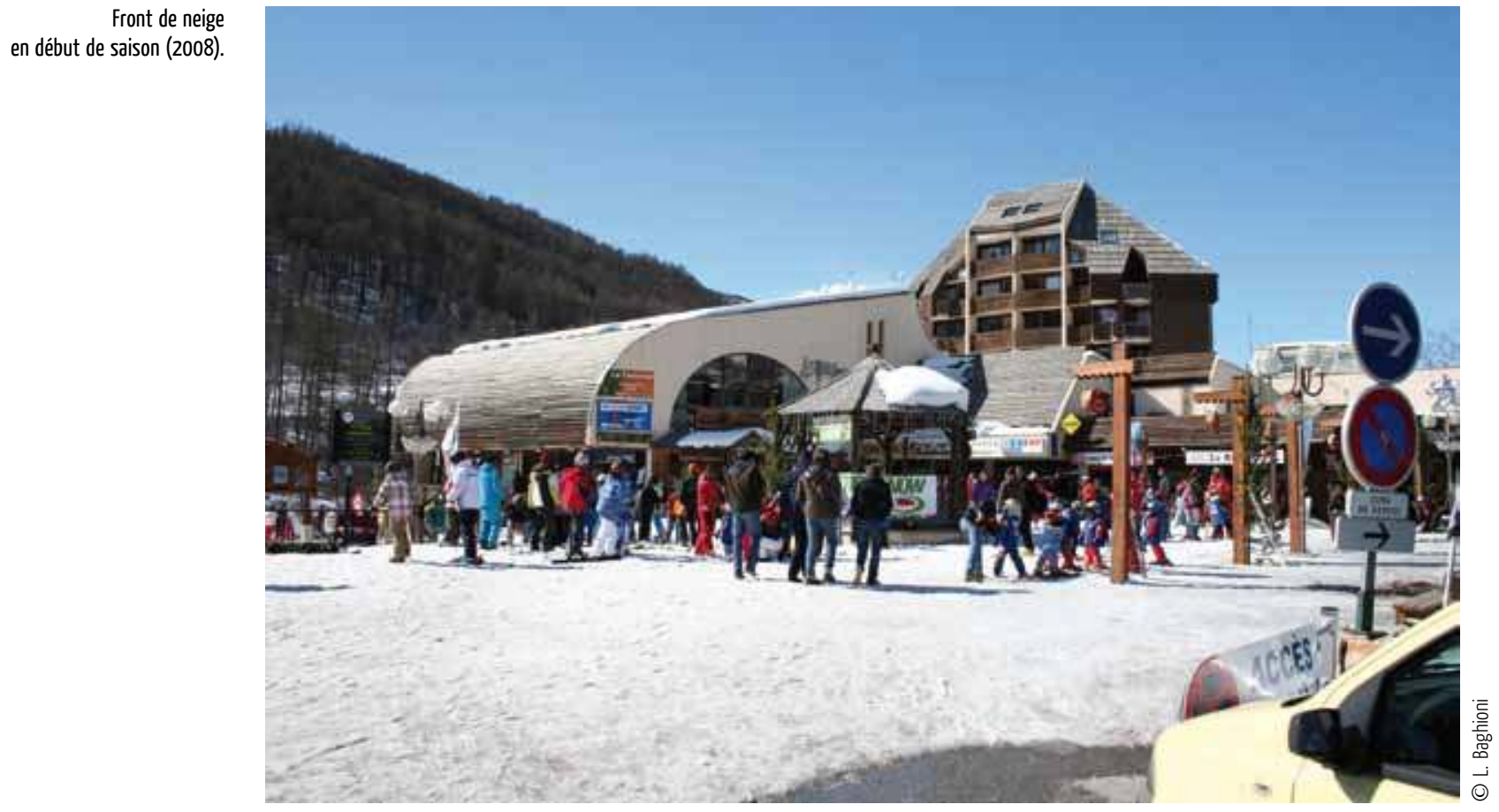


vacanciers, encore faut-il dévoiler le fonctionnement concret de ce mécanisme, dans les entreprises et les lieux de vie de ceux qui en font l'expérience. À ce titre, cet article entend montrer que l'analyse des modes d'habitat pratiqués par les saisonniers du tourisme peut être une entrée féconde. Il s'agit de mettre en œuvre une ethnographie qui ne se cantonne pas aux interactions clients-employés, mais qui tente de saisir la place des saisonniers dans l'entreprise sans la dissocier des conditions d'existence de ces travailleurs.

\section{Des saisonniers du tourisme dans les montagnes françaises}

Dans les années 1960 la massification du tourisme et l'avènement de nouvelles pratiques de vacances ont refaçonné la morphologie des territoires de montagne français. Ces changements ont été largement impulsés par le Plan Neige de l'état (1964) qui visait à aménager des stations nouvelles, fonctionnelles et performantes. Avec le déploiement de l'activité touristique, des emplois saisonniers se sont développés. Mais l'engouement qui accompagne alors l'essor des sports d'hiver relègue au second plan les problématiques liées au logement des salariés. Jusque dans les années 1990, les nombreuses études commandées par l'État se concentrent avant tout sur l'accueil des vacanciers (Wozniak 2002); le logement de la main d'œuvre saisonnière y est quant à lui très peu pris en compte ${ }^{3}$. Simultanément, en station, le prix du foncier augmente et devient prohibitif pour les salariés saisonniers, surtout les moins qualifiés.

Alors que la question du logement des travailleurs demeure impensée, le rythme saisonnier dans le secteur touristique s'institutionnalise. À la différence de l'activité agricole, les temps de travail sont déterminés par l'accueil des touristes, principalement en été et en hiver, laissant vacantes des périodes de 2 à 3 mois d'intersaison, en automne et au printemps. Dès lors, les saisonniers du tourisme rejoignent les rangs de l'intermittence de l'emploi. Selon Annie Lamanthe (2008), jusque dans les années 1980, l'incertitude liée aux variations saisonnières de l'activité est partiellement régulée par une relation salariale de type "paternaliste ${ }^{4}$. Celle-ci permet notamment de conjuguer « la flexibilité avec une grande stabilité » (Lamanthe 2008 : 29), en offrant un certain nombre d'avantages aux employés saisonniers (dont le logement) en contrepartie de leur fidélité et de leur subordination. Dans la décennie suivante, une certaine « distanciation, formalisation et juridicisation des relations de travail révèlent l'effritement du "compromis salarial" et la manière dont se transforment les sources, les formes et les statuts de la flexibilité » (ibid.).

La question du logement rejoint ici celle des statuts sociaux actuels des saisonniers. « Population jeune, essentiellement masculine, relativement peu qualifiée et aux profils professionnels variés ${ }^{5}$, les saisonniers sont affiliés à un régime d'emploi équivoque. Les Contrats à Durée Déterminée Saisonniers ne sont pas soumis à des clauses de reconduction du contrat de travail ${ }^{6}$. La prime de précarité de fin de contrat, qui existe pour d'autres catégories de " précaires » (intérimaires, par exemple), est absente de la législation contractuelle de cette main d'œuvre. De plus, la succession de deux contrats en CDD Saisonnier donne suite à l'application d'une assurance chômage à coefficient réducteur ${ }^{7}$. Il faut ajouter à cela l'importance du travail non déclaré dans les stations touristiques, principalement dans l'hôtellerie-restauration (Dethyre 2007). 


\section{Du logement ...}

Dans ce contexte, les saisonniers disposent de trois principales solutions de logement. La première possibilité est la colocation chez un particulier. Cette démarche entraîne d'une part des problèmes de versement de la caution et du premier mois de loyer qu'il leur faut payer avant le début du contrat de travail; d'autre part, le bien loué doit se trouver relativement proche du lieu de l'activité salariée, étant donnés les problèmes de déplacements liés aux chutes de neiges hivernales. Cependant le prix du foncier en centre-station a tendance à repousser l'installation en périphérie, dans les alentours. La seconde éventualité est celle de l'habitat mobile en camion. Dans ce cas-là, les difficultés d'établissement seront d'autant plus prégnantes: ces véhicules ne sont pas autorisés à stationner plus de 48 heures sur le même emplacement dans les communes de montagne où l'on peine à trouver des aires aménagées et peu coûteuses. Les premiers arrivés parviendront à se rabattre dans les campings. Néanmoins, ces établissements n'acceptent généralement pas plus de 3 ou 4 camions qui devront stationner sur les emplacements les moins visibles, à l'abri du regard des touristes ${ }^{8}$. La dernière solution consiste à trouver un poste où l'employeur propose un logement. Système comparativement plus pratique que les autres, il évite l'étape de la recherche d'un appartement et le plus souvent celle du paiement du premier loyer (les loyers sont directement retirés sur les paies). Le saisonnier emménage alors soit dans le bien privé de l'employeur, à l'écart du lieu de travail, soit sur les lieux mêmes de l'activité salariale. Cette «formule » reste quantitativement résiduelle mais demeure largement valorisée par les institutions et associations locales qui la présentent comme « une chance » en comparaison aux autres modes d'habitats disponibles ${ }^{9}$.

Si l'on se penche maintenant sur le discours des saisonniers, les représentations et usages de ces divers logements s'avèrent plus complexes à saisir. Pour les travailleurs mobiles, cette forme d'emploi engage à occuper plusieurs logements ou, tout au moins, à parcourir et à s'installer temporairement sur différents territoires. Entre périodes passées dans la maison familiale ou en colocation, dans un appartement en ville ou à la montagne, seuls ou entre pairs, les mobilités recèlent leur lot de stratégies et d'organisation structurées par les temps d'activités salariales et les périodes chômées. L'habitat évoque par là des rapports de complémentarité (Jeanjean 2011 ; Sénépart 2011) : entre territoires et types d'activités de travail exercées. Autrement dit, les façons de se loger en saison peuvent correspondre à différentes formes professionnelles. On constate, par exemple, que le mode «nourri-logé » est plus fréquent dans la restauration pour les nouveaux candidats à la saisonnalité, et qu'il est plus rare pour les saisonniers qui possèdent une bonne connaissance du territoire. L'analyse des modes d'habiter de ces travailleurs se révèle ainsi pertinente pour penser l'articulation entre mobilités et phases de la «carrière » (Hughes 1996).

Les différents types de logements présentés plus haut ne pourront évidemment pas être abordés dans un seul article. Mais il en est un qui retiendra notre attention: le mode «nourri-logé ». Nous allons maintenant nous pencher sur le cœur du problème: les pratiques habitantes, ici inscrites entre contraintes économiques et injonctions à l'invisibilité. 


\section{... À l'habitation}

Au début du xxe siècle, Marcel Mauss encourageait les ethnologues à se pencher sur « la manière dont la forme matérielle des groupements humains, c'est-à-dire la nature et la composition de leur substrat, affectent les différents modes de l'activité collective » (Mauss 2006 [1950] : 390). L'auteur pointait l'importance de l'étude des modes d'habitat humains articulée à celle des rythmes collectifs de la vie sociale. De ces travaux découle l'idée que l'habitation ne peut être complètement séparée des autres sphères de la vie des individus, elle est une «modalité nécessaire de l'existence sociale » (Bonnin 1994 : 109). Il convient donc de ne pas s'en tenir à la notion trop restrictive de « logement ${ }^{10}$ pour se rapprocher de celle d'habitation ${ }^{11}$, plus apte à explorer les dimensions matérielles de l'habiter et les logiques ancrées dans le quotidien des sujets.

Par ailleurs, les modes d'habiter renvoient à des rapports de pouvoirs et de domination. Cette dimension politique des espaces construits a été maintes fois mise en exergue, et notamment dans les écrits de Michel Foucault (1975). Cette orientation incite à explorer les répercussions d'une organisation spatiale sur la façon dont les sujets se l'approprient... ou peinent à le faire. Ce parti pris s'avère pertinent pour penser l'espace social à fortes contraintes qu'est le centre de vacances dans lequel vivent les saisonniers « nourris-logés ».

Il s'agit donc de proposer l'ethnographie d'un lieu qui regroupe habitation et travail. À ce propos, et pour ce qui est du cas français, de nombreux auteurs insistent sur la perméabilité entre sphère productive et domestique (Weber 1986; Monjaret 2001; Flamant $\&$ Jeudy-Ballini 2002). Ces analyses laissent toutefois apparaître que la norme dominante demeure celle d'une frontière spatiale, marquée par des passages (portes, rues, trajets divers) qui provoquent le changement de lieu. Ainsi, la situation qui consiste à vivre à l'endroit même où prend place le travail provoque un certain nombre d'interrogations. Comment les saisonniers s'adaptent-ils à ce mode d'habitat qui questionne l'idée d'autonomie associée à la notion d'habitation occidentale, tandis que celle-ci est à la fois sur les lieux du travail et soumise au regard hiérarchique? Dans ce cadre, comment est pensé le hors travail, et plus particulièrement le « chez-soi », pour ces mobiles «nourris-logés »? Enfin, que révèle l'ethnographie de ce mode d'habiter à propos de la place accordée aux saisonniers dans l'entreprise, mais aussi de l'usage de ce type d'habitation dans la carrière des saisonniers?

Le propos suivra un développement chronologique, du début à la fin de la saison d'hiver, afin d'en esquisser son déroulement. Les données qui seront utilisées ici émergent d'un terrain mené par observation participante, qui s'est étalé sur la saison 
d'hiver 2008-2009 durant 4 mois et demi. Pour cette enquête en immersion dans un « village vacances » du Sud Est de la France (05), j’ai été embauchée à temps plein, comme serveuse, nourrie-logée sur mon lieu de travail.

Dans un premier temps, je livrerai quelques éléments descriptifs à propos du centre de vacances dans lequel a été menée l'enquête. Dans un second temps, j'examinerai les rapports de pouvoirs qui se mettent en place au sein de l'établissement. On verra comment les interférences entre sphère privée et sphère publique, entre intimité et contrôle social, donnent lieu à la mise en place de mécanismes de distinction qui prennent leur source dans ce que chacun décide de faire de son temps libre. Un temps libre qui n'en reste pas moins un temps à occuper et dont l'usage qui en est fait se répercute sur la perception de soi et des autres. Enfin, on évoquera un temps autre, celui du retour, qui annonce l'intersaison, afin d'insister sur les liens de continuité ou de rupture avec d'autres types de pratiques habitantes.

\section{Au centre de vacances}

Pour les employés «nourris-logés », comme cette dénomination l'indique, certaines activités domestiques s'effectuent sur les lieux de travail: manger dans l'espace du restaurant (trois repas par jour avec l'impossibilité de réaliser sa propre cuisine et donc l'obligation de se soumettre aux mêmes menus hebdomadaires pendant 4 mois et demi), dormir, faire les lessives, consulter Internet, etc. Les espaces du bar, du restaurant, de la buanderie, de l'ascenseur, des couloirs qui mènent aux chambres ainsi que l'extérieur de l'établissement, sont à partager avec les clients et les autres employés. Aucune salle,

Le « village vacances » dans lequel a été menée l'enquête n'est en réalité qu'un seul établissement pouvant accueillir un peu plus de 150 clients qui disposent sur place d'un certain nombre de prestations

(animations pour adulte et enfant, restaurant, bar, hôtel, salle de jeux, solarium, buanderie, salle de spectacle, parking). L'immeuble est encerclé d'une barrière qui matérialise un espace privé. Ce bâtiment ne possède qu'une seule entrée qui donne directement sur l'accueil (2008).

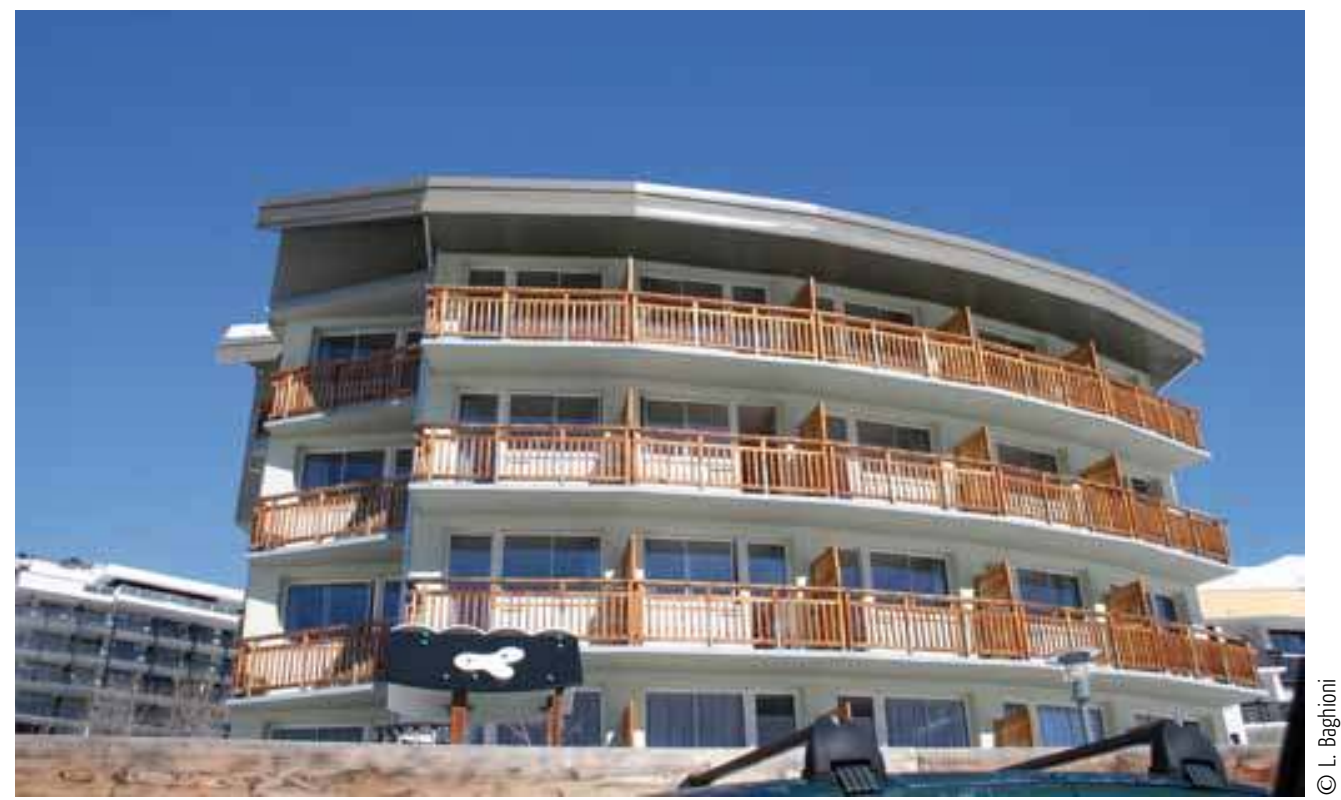




\begin{tabular}{|l|l|l|l|l|}
\hline Locaux/mobiles & Type de logement & Répartition & Âges & Postes de travail \\
\hline Locaux & $\begin{array}{l}\text { Logés dans les villages } \\
\text { alentours }\end{array}$ & 11 & Entre 24 et 48 ans & $\begin{array}{l}\text { Accueil, responsable anima- } \\
\text { tion, chef de cuisine, respon- } \\
\text { sable de l'entretien, femme de } \\
\text { ménage. }\end{array}$ \\
\hline Mobiles & $\begin{array}{l}\text { Nourris-logés dans le } \\
\text { centre de vacances }\end{array}$ & 7 & Entre 20 et 30 ans & $\begin{array}{l}\text { Serveuses, aide-cuisiniers, } \\
\text { animateurs, plongeurs. }\end{array}$ \\
\hline Mobiles & $\begin{array}{l}\text { Logés proche du } \\
\text { centre-station }\end{array}$ & 1 & 28 ans & Animatrice enfant \\
\hline
\end{tabular}

aucun espace collectif ne sont réservés aux saisonniers. Les chambres qui leur sont allouées en début de saison ressemblent à celles d'un client. Cependant, ce sont les moins bien exposées, les plus petites et les moins bien chauffées; il est également possible que certains aménagements y fassent défaut (prise télé, meubles supplémentaires etc.). Ces lieux privés, d'une surface de 11 à 14 mètres carrés, sont composés d'une salle de douche et d'une pièce largement occupée par un lit et un placard.

Dans les centres hôteliers de ce type, les périodes de forte activité et les cadences du travail sont comparables d'un établissement à l'autre. Commençons par la description du rythme quotidien et des scansions journalières. La majorité des employés, qu'ils appartiennent à l'équipe de la restauration, à celle de l'animation, de l'accueil ou de l'entretien travaillent sur le mode de la « coupure $»^{12}$, c'est-à-dire que leur journée de travail, à un moment ou à un autre, selon le secteur, s'interrompt quelques heures pour reprendre ensuite. Se libèrent ainsi certaines plages horaires de temps libre, de 3 à 4 heures, limitées par la reprise du travail. Les congés hebdomadaires, quant à eux, durent un à deux jours et réunissent ou disjoignent les employés des différentes équipes.

Si l'on change maintenant d'échelle temporelle pour se placer sur la période globale de la saison, on peut dire que les saisonniers la découpent en trois grandes phases. Le début de saison est un moment de découverte ou de (re)démarrage; c'est un temps où les équipes de travail se mettent en place afin de se soumettre au «test du mois de décembre » et des vacances de Noël: les employés sont alors en période d'essai, si cette quinzaine « se passe mal », ils seront aussitôt remerciés puisqu'il est alors considéré qu'ils ne seront pas aptes à « tenir » toute la saison. Les saisonniers candidats sont alors jugés autant sur leurs compétences relationnelles (avec les clients et les autres employés) que techniques (dextérité et initiative individuelle pour l'exécution des tâches au travail). Autre temps, celui de la pleine saison (février), il représente l'apogée de l'intensification du travail. Un travail devenu routinier (les lieux et tâches sont alors connus) qui s'accompagne de nombreux conflits intra ou inter équipes. Pour finir, la fin de saison, qui recouvre le mois de mars et avril, période durant laquelle le travail devient plus calme, on y voit poindre les questions relatives à l'après saison.
Répartition des employés saisonniers du centre de vacances en fonction du type de logement occupé, de leur âge, et de leur poste de travail. 20 employés travaillent au sein du village vacances, dont 19 saisonniers. 7 d'entre eux sont nourris-logés sur place. 


\section{Ambiguité du contrôle hiérarchique}

Au mois de décembre, lors de la réunion d'accueil des salariés, la directrice insista sur diverses règles à observer pour que « la saison se passe bien ». Fut d'abord exprimée l'interdiction de rester dans les espaces occupés par les clients en dehors des heures de travail, ainsi que la recommandation d'éviter tout rapport trop familier avec les clients habitués. La suite des prohibitions concerna l'occupation des chambres: ne rien poser sur le rebord des fenêtres dans le but de s'en servir de réfrigérateur, ne pas fumer ou boire de l'alcool, ne pas faire de bruit et, de manière générale, ne pas favoriser les conflits divers en étant trop proches au sein des équipes, ce qui pourrait nuire à l'ambiance de travail. La longue évocation de ces règles était significative de la redondance de problèmes rencontrés lors des saisons précédentes. Mais surtout, elles posaient d'emblée, au grand jour, la surveillance dont feraient l'expérience ceux qui seraient nourris-logés sur le centre. "On se croirait à l'internat », glissa l'un d'entre eux lorsque le discours de la directrice fut terminé. Chacun compris qu'il était dès à présent dans une situation fort ambiguë, soumis à une autorité qui dépasserait les cadres du travail. Pourtant pas élève mais employé, pas non plus prisonnier mais sujet pris dans le regard hiérarchique jusque dans son intimité.

Les règles prescrites lors du début de saison ne seront observées que durant une courte période s'étendant de la mi-décembre à la première semaine de janvier. Les deux saisonniers mobiles les plus anciens avertirent les autres: " au début faut rien faire, faut être tranquilles, comme ça après elle fait moins attention ». La tactique consiste à se « fondre dans le moule », dans les premiers temps de la saison, autant dans les activités menées dans les chambres que dans celles exercées au travail. La sphère privée et celle du travail s'informent l'une, l'autre: il faut être exemplaire des deux côtés pour ne pas attirer l'attention.

L'employeuse, quant à elle, se trouve également à la jonction de deux univers habituellement distincts. Elle énonce des règlements à observer en dehors du travail, afin de tenir secrètes les coulisses de son établissement. Mais sa légitimité à les faire respecter est mise en porte-à-faux par la nature même de ces règlements qui atteignent l'intimité des individus. Sa pratique de l'autorité hiérarchique est dès lors délicate. Elle doit se maintenir informée du comportement hors travail de ses employés, « repérer les rapports potentiellement problématiques, "les clans » et veiller à ce que leurs vies privées dans le centre ne prennent pas le pas sur le travail», comme elle l'expliqua au cours d'un entretien. Il s'agit d'empêcher ses employés de se comporter comme « chez eux » dans des espaces dans lesquels ils sont susceptibles d'êtres vus par les clients. Tout se passe comme si les rapports entre employés ne devaient pas trahir la priorité du lien à l'employeur et au travail $^{13}$. Néanmoins, la directrice reste confrontée à la méconnaissance des logiques qui œuvrent en partie sans elle, en dehors du travail (elle ne vit pas au centre), et qui participent largement à construire les rapports au travail.

\section{Adaptation à l'espace-temps du centre: promiscuité et travail}

Les regards s'immisceront dans les plus petits espaces de la vie quotidienne et seront principalement entretenus par les collègues de même rang hiérarchique. Cette vigilance 
mutuelle entre employés, favorisé par la disposition et la mauvaise insonorisation des chambres (qui sont côte à côte ou l'une au dessus de l'autre), vise en premier lieu à repérer son voisin. Les comportements sont alors habilement mesurés afin de s'assurer que le non-respect des règles ne sera pas divulgué à la hiérarchie. Ces observations réciproques sont une présence perpétuelle qui montre à l'autre que l'on sait; que l'on sait s'il est sorti du centre puisqu'il a été vu à l'accueil, que l'on sait s'il a téléphoné la vieille au soir et quelle conversation il a eu, que l'on sait s'il stocke de l'alcool ou d'autres denrées sur sa fenêtre, s'il est allé laver son linge puisque les machines étaient toutes occupées, que l'on sait qu'il n'était pas dans sa chambre tel jour à telle heure puisque les coups portés sur sa porte sont restés sans réponse, que l'on sait à quelle heure le trouver aux consultations internet, au restaurant pour le repas, etc. Même dans l'espace privé, la solitude s'éprouve difficilement.

Une fois la période de repérage et de classification de l'autre terminée, les «nourrislogés » mettent en place diverses tactiques d'évitement. Pour cela, il a donc fallu que passe un certain temps d'observation et que se réalise l'appropriation de l'espace, « victoire du lieu sur le temps », selon la définition de Michel De Certeau (1990 : 60). Certains endroits seront ainsi privilégiés ou évités, selon les moments de la journée ou les emplois du temps de chacun. Prendre l'escalier plutôt que l'ascenseur pour aller étendre son linge en fin d'après-midi, téléphoner dans la salle de douche qui est davantage éloignée de la cloison qui sépare les deux chambres, envelopper de plastique opaque tout ce qui est placé sur la fenêtre; ou dans un autre registre, éviter de côtoyer les collègues de travail durant son jour de congé. À ce titre, l'exemple de Jennifer est éloquent. Jeune femme de 26 ans, « nourrie-logée » au centre, elle quittait sa chambre pour rejoindre les lieux de travail durant ses jours de repos. Elle choisissait précisément l'heure où elle pourrait trouver untel ou unetelle et déclarait pourtant sur le ton de l'humour: « Bon, je suis passée, même si normalement on doit pas se voir aujourd'hui, j'ai pas respecté l'intervalle réglementaire ». De son côté, lorsque je questionnais Raphaël, animateur, à propos de sa vie au centre, celui-ci me rétorquait «Ici on ne se fait pas vraiment de chez-soi. C'est pas possible. Déjà parce qu’on sait dès le départ qu’on va partir et en plus parce qu’on est épié de partout ».

S'il est vrai qu'en tout début de saison le temps n'a pas encore agi sur la proximité qu'entretiennent les saisonniers entre eux, les relations tendent à s'intensifier à une vitesse surprenante, accumulant le temps passé ensemble au travail et la vie collective du centre, elles se construisent à un rythme singulier, défiant les normes communément admises; le collègue de travail finit par s'immiscer dans chacun des temps libres des individus. «Lorsque le territoire de l'intimité se construit sur le modèle d'une propriété partagée, les relations entre les membres ont un caractère fusionnel », avance Alain Thalineau (2002 : 42-43). Dans ce cadre, les groupes se forment sur la base d'un contrôle possible du temps de l'autre. Autrement dit, ceux qui ne sont pas logés à la même enseigne, les «locaux», seront écartés de certaines activités extérieures au travail ou des réseaux d'échanges de livres et films pour occuper les temps morts, par exemple. Pour les locaux, la frontière entre travail et hors travail est moins ténue, principalement parce qu'ils contrôlent davantage le regard qui sera porté sur leur intimité. Si les locaux peuvent se vanter d'avoir une «vie en dehors du travail », comme ils aimaient à le répéter parfois avec ironie, ils soulignent par là la construction, dans la sphère privée, d'une relative stabilité face aux rythmes des saisons (certains sont mariés, ont des enfants, ont établi des liens de fidélité avec leur employeur). Par contre, les mobiles ne connaissent pas ou peu le territoire et ne possèdent pas de réseaux de sociabilité autres que ceux du travail. Il ne s'agit pas de dire qu'il est 
impossible, pour les «nourris-logés», de développer des liens à l'extérieur du centre, mais ces réseaux là prendront davantage de temps.

Au centre, tout se passe comme si les sujets recréaient des relations suffisamment fortes pour être assimilées à des connaissances de longue durée, des rapports familiers. Les uns ne font plus rien sans les autres et avant chaque coupure chacun se renseigne sur l'activité prévue. Les instants de solitude se raréfient au fur et à mesure que le temps s'écoule de sorte que, finalement, celui qui esquive les activités collectives soit perçu comme méprisant. Cela entraînera, sauf s'il fait preuve de «bonnes raisons », des attitudes pénalisantes (on manquera par exemple de le tenir au courant lors d'une prochaine sortie de groupe qui viserait à éviter d'ingurgiter la nourriture du centre). S'impose doucement l'obligation de se raconter, de dire ce que l'on fait, qui l'on est. C'est que cette sociabilité dense pallie un manque, celui d'un espace et d'un temps maîtrisable, propre à l'univers de «la maison» (Leroi-Gourhan 1964 : 140). Cet espace-temps spécifique révèle ainsi à quel point l'habitat ne se réduit pas à la seule dimension matérielle du logement et comment les individus s'efforcent de créer des liens pour rendre l'espace vivable et s'intégrer ainsi dans un environnement dans lequel ils se façonnent sécurité et certitude (Pétonnet $2002:$ 43).

Dans cette situation, qui entremêle si étroitement travail et hors travail, l'autre, celui que l'on côtoie chaque jour, représente un soutien permanent pour «tenir la saison » jusqu'au bout. Mais il est aussi un référent à l'univers du travail contraignant, lieu de tous les conflits. Le collègue finit par être tout autant sécurisant qu'envahissant. C'est donc une perception sous-jacente du temps et du lieu de vie qui est révélée, une mesure subjective qui vise à trouver la « bonne distance », pour reprendre une formule de Denis Guigo (1991). Un équilibre entre intimité et travail, évitement des autres ou besoin de leur présence palpable: l'analyse des discours et pratiques dans le hors travail révèle la construction de toute une économie temporelle de l'existence sociale en saison. Celle-ci est ainsi ancrée dans la confusion des sphères privées et publiques, largement relayée par l'espace pourtant clos des chambres.

\section{Les chambres: un lieu à soi?}

Lors de la visite des chambres, je me suis aperçue qu'aucun de mes compagnons ne les avait décorées à leur goût, y laissant même les tableaux prévus pour les touristes dont chacun trouvait à se moquer. Objets «étrangers», ceux-ci représentaient une incursion acceptée dans l'espace standardisé mais néanmoins privé de la chambre d'hôtel. « J'ai apportéle strict minimum » me commentait Anthony, plongeur, en me montrant ses affaires, "le snow, la TV et la console de jeux. Ah oui, et des vêtements pour le travail quand même ". Bien souvent, les effets personnels sont rassemblés à un seul endroit, proche du lit, ultime lieu de l'intimité et du repos des corps. Les rares objets ramenés le sont d'abord dans un souci de se raccrocher au monde extérieur lorsque l'ambiance du centre est trop pesante: c'est le cas de la télévision, des équipements de sport d'hiver qui autorisent l'évasion de l'espace du centre, ou encore de la photo du conjoint qui renvoie à l'univers des proches.

L'espace privé de la chambre est visible du dehors et audible d'à côté. La chambre n'en demeure pas moins un espace clos, mais reste soumise aux incursions diverses de 
la sphère du travail. Incursions via le système d'aération, qui laisse pénétrer les odeurs de nourriture du restaurant, qui s'infiltrent et envahissent la chambre. Les odeurs sont ainsi d'autant plus redoutées durant les jours de congé. Elles paraissent anéantir l'opacité des cloisons, rappelant l'activité de travail incessante, en train de se dérouler quelques mètres plus bas ${ }^{14}$. Incursions également personnifiées par les « collègues » qui se trouvent précisément mêlés à ces deux versants de la vie quotidienne du centre. Nous l'avons vu, ces derniers possèdent les clés pour exercer un regard panoptique (connaissances des emplois du temps, des personnes qui occupent des postes privilégiés d'information comme celui de l'accueil, etc.). Il est donc bien difficile de repousser les visites qui rythment les coupures de travail et qui défont le désordre que chacun s'autoriserait si personne ne devait « passer » sans en avertir l'hôte.

S'isoler n'est pas pour autant complètement impossible. Mais s'isoler nécessite des réarrangements territoriaux et relationnels qui dépendent tout à la fois des contraintes de ce type d'habitat (une pièce avec salle de douche) et des temps d'occupation de celuici. De cette situation émerge une impression partagée. Du travail, en pleine saison, les «nourris-logés» le disent chaque jour, ils ne parviennent pas à s'en décrocher. Les frontières se brouillent, et si le travail est communément considéré comme une sphère où règne la collectivité, la sphère privée, quant à elle, n'est pas considérée comme telle.

L'impression d'« enfermement ", dont feront part ces saisonniers, résulte aussi de la peine éprouvée à marquer une distinction suffisante entre travail et hors travail. Le sentiment qui prédomine et qui est maintes fois exprimé est alors celui d'être coincé dans un présent répétitif, dans « une bulle, hors réalité » (Myriam aide-cuisinière, 20 ans). Le lieu de travail et le lieu de vie œuvrent ensemble comme le «module d'enfermement » que décrit Michel de Certeau (1990 : 165), désignant par là « une bulle du pouvoir panoptique et classificateur, [...] un espace qui rend possible la production d'un ordre autonome ».

En pleine saison, il est par ailleurs significatif de repérer qu'aucun employé ne parle de la fin de saison. Le temps social dominant, extérieur à la station touristique, est comme suspendu, provisoirement nié, tant le travail en saison est à contretemps. Seul le présent semble compter, les saisonniers sont alors tous tournés vers l'investissement des plus petites séquences temporelles, des temps libres qu'il s'agit de combler. L'objectif est de ne pas trop penser puisqu'évaluer le temps qui passe, à ce stade, provoque l'ennui ou l'angoisse. "Là c'est la
Même pendant les jours de congé, les lits sont refermés pour produire le " passage de dans le lit" à" sur le lit" » (Rosselin 2002 : 108), afin de maintenir le caractère personnel de ce meuble, transformer le lit en canapé et recevoir d'autres personnes grâce à ce geste précis (2009).

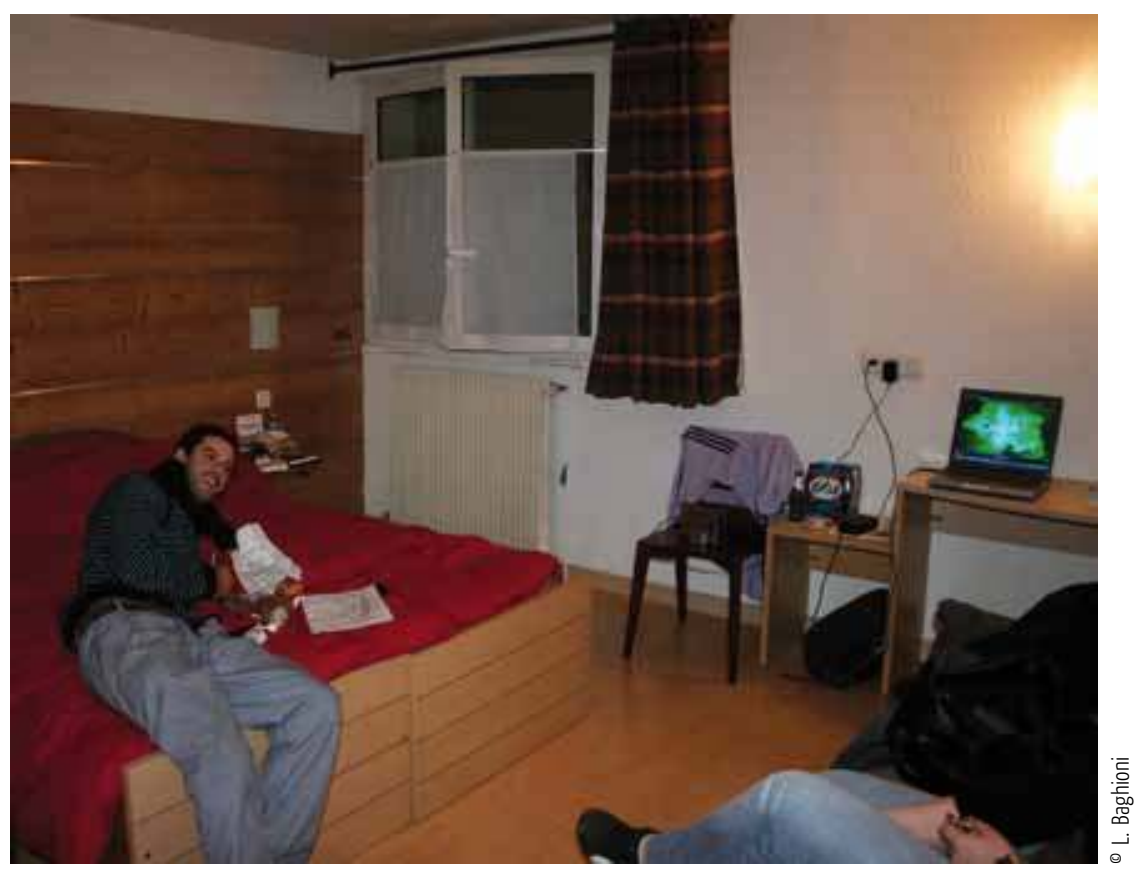


période où faut que je fasse un truc tous les jours, que je sorte, pas envie de rester dans ma chambre, avec le rythme au boulot, sinon je deviens fou », confie Anthony. C'est alors l'extérieur du centre qui représente le territoire le plus prisé. Il faut sortir de ce lieu, s'en éloigner un instant.

\section{Les conflits: distinction et légitimation de soi}

Espaces protégés ou conquis, les lieux du travail restent unis par la règle de la « satisfaction du client ». En coulisse, les conflits jalonnent le quotidien, mais ne doivent jamais être exposés sur le devant de la scène. "Le client » pousse à un certain retranchement de soi, et modèle des attitudes de «façade ». Il est aussi convoqué pour asseoir l'importance et les valorisations que chacun se fait de sa propre activité. Dans ce cadre, la coopération s'établit sur un mode conflictuel, mais les objectifs fixés par l'organisation du travail sont toutefois maintenus. Les « réconciliations » se font durant les conversations hors travail. Celles-ci s'imposent comme de véritables défouloirs face à la pression de l'exécution des tâches et à la tentation, toujours présente, d'un relâchement, qui équivaudrait à prendre appui sur son collègue devenu ami. Les pratiques de « braconnage temporel » (De Certeau 1990 [1980]) ${ }^{15}$ se réalisent principalement en équipe, leur exercice solitaire entraînant l'exclusion des rapports amicaux qui permettent de « tenir » la saison. De cette manière, les conflits du travail sont étonnamment acceptés et font office de norme. " $L a$ saison c'est comme ça, surtout en février, tout le monde se prend la tête », lançait souvent le chef de cuisine. Le rapport au temps de travail global qu'est la saison est ici déterminant: chacun sait jusqu'à quelle date se maintiendra cette situation. Situer très précisément l'arrêt du travail est ici une condition qui structure le rapport aux contraintes, aux conflits, aux autres. Les remarques colériques apparaissent aussi vite que leur pardon. Et cette attitude est considérée comme un trait de caractère qu'il faut posséder pour bien vivre la saison.

Malgré cela, certaines distinctions apparaissent comme irréductibles et trouvent leur source dans les occupations que chacun met en œuvre dans le hors travail. Elles tendent à relier étroitement deux pendants de la vie en saison: les attitudes au travail et le rapport avec le lieu de la station touristique de montagne. Les pratiques au dehors façonnent les affinités au dedans. Schématiquement, les processus de différentiation divisent les saisonniers en deux groupes, ceux qui profitent du cadre naturel et ceux qui sont supposés ne pas (assez) le faire. En effet certains mettaient un point d'honneur à skier ou faire des randonnées toutes les fois que le temps le leur permettait. Le fait de ne pas profiter de la montagne apparaissait pour le groupe « des sportifs » comme une attitude absurde, d'autant plus que pour eux (qui possédaient une plus longue expérience des saisons), les sorties régulières dans les bars, les journées passées à faire du shopping ou dormir, représentaient un signe flagrant d'une méconnaissance des règles tacites admises par les saisonniers. « Ça, sortir comme ça et dépenser ta paie dans les bars, tu le fais une saison pas plus, après tu t'aperçois que sinon tu claques tout l'argent que t'es venu gagner, c'est n’importe quoi », me disait Raphael qui, de son côté achetait chaque mois un équipement de ski coûteux pour s'adonner à sa passion. Cela nous permet de remarquer que ce n'est pas tant la dépense qui le gêne, que le type de dépense, bien « durable » ou « dilapidation ». « Ici, si t'es comme ça t'es vite catalogué. Si ton but c'est de revenir faire la saison tu ne peux pas te permettre et faut apprendre la discrétion aussi », commentait Yazid, employé à l'accueil depuis 10 ans 
et sédentarisé dans un village proche de la station. De leur côté, celles et ceux qui refusaient les activités en lien avec le milieu naturel manifestaient aussi beaucoup d'incompréhension. C'est le cas de Jennifer qui faisait sa première saison en montagne et commentait: "Qu'est-ce que ça peut leur faire si je ne fais pas comme eux pendant mes repos, moi la montagne le ski tout ça je m'en fiche, ça ne me plaît pas, on dirait que quand tu travailles ici tu n'as pas le droit de penser ça ». On note ainsi que, pour ceux qui adhèrent aux valeurs qu'ils construisent comme étant celles du milieu montagnard, les activités réalisées en dehors du travail représentent un « profit symbolique » pour reprendre la terminologie de Pierre Bourdieu; elles inscrivent les individus dans un rapport différentiel au travail saisonnier et à son environnement.

Ces mécanismes révèlent donc une certaine posture visà-vis du travail saisonnier en montagne mais ils sont également des indicateurs de la variété des rapports à l'avenir et à la carrière. L'occupation des temps libres et les types de consommation qui y sont accolés sont des moyens, visibles, palpables, permettant de repérer ceux qui ne font que passer et s'y confronter. C'est que, pour ceux qui s'installent dans cette forme d'emploi, le fait d'être soumis à une rythmicité perçue comme naturelle - celle des saisons - est construit comme un rapport positif au monde, une manière de donner du sens à cette condition de saisonnier, en dehors des contraintes inhérentes à ce mode de travail.
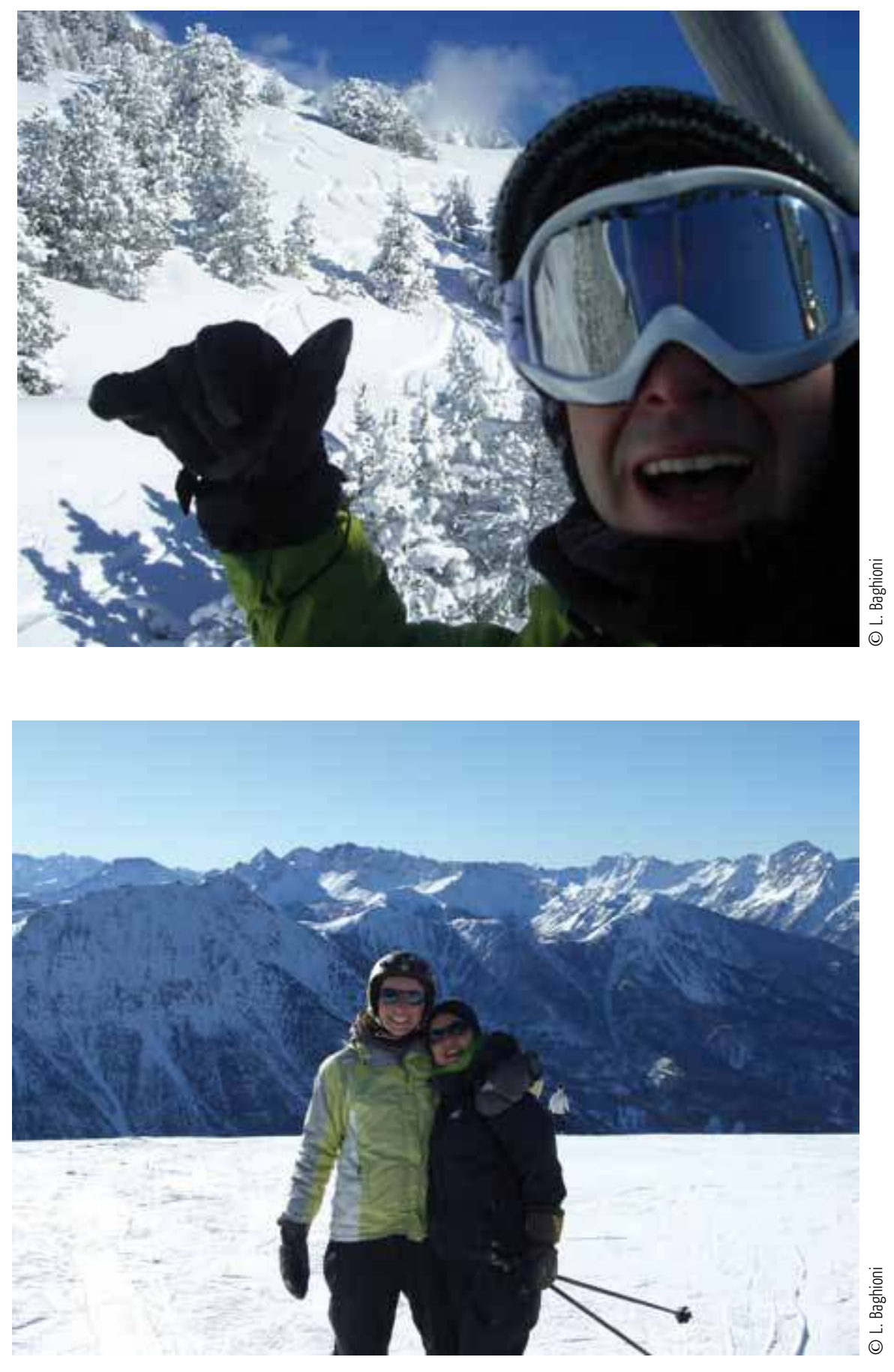

Photographies types d'un « souvenir de la saison » (2009). 


\section{Le temps du retour}

En fin de saison, le rythme du travail ralenti. Les conflits sont alors quasi inexistants et provoquent maintenant des attitudes de résignation tranquille. Les aspirations des travailleurs se tournent vers l'après-saison. Parler d'un retour « chez-soi » fait alors émerger les raisons qui ont provoqué le départ: chercher du travail, fuir un ex-conjoint ou un quotidien dépourvu de sens; ou enfin, des ruptures d'ordre familial. Les liens passés, qui étaient mis en sourdine durant la saison, réapparaissent. Selon les parcours de chacun, les saisonniers sont soit face à la peur des retrouvailles avec ce qui avait été fuit, soit dans un « quant à soi $»^{16}$ à nouveau conquis, dans lequel les projets individuels peuvent être privilégiés. C'est la proche satisfaction d'un temps finalisé. Le prix a été payé pour vaquer à d'autres occupations. De cette manière, la fin du travail est en mesure de réactiver l'ambition et l'illusion d'une existence « uniquement » pour soi. Les projets d'achats de restaurant, de tours du monde ou de bifurcation vers des études longues sont proférés sans que des solutions pratiques pour la mise en œuvre de tels desseins ne puissent être seulement devinées.

Les espérances se centrent aussi sur ce qui a été laissé en l'état, au moment du départ. Le retour est souvent une confrontation au vide (absence des collègues de travail et des activités quotidiennes liées à l'emploi) et à un sentiment d'étrangeté dans un cadre qui est pourtant bien familier: la maison parentale ${ }^{17}$. Loger dans l'espace du foyer familial est alors vécu comme une régression, économiquement nécessaire, mais potentiellement conflictuelle. C'est le constat d'un impossible retour à l'identique, d'un décalage avec les proches. C'est aussi un retour vers une temporalité dominante de laquelle le temps de la saison avait écarté ces travailleurs. Indéterminations face à l'avenir, mise en place d'une stratégie pour la saison prochaine afin de mettre provisoirement un terme à cette anxiété, les journées sont alors scandées par les justificatifs administratifs de tout ordre (changement d'adresse, rendez-vous au Pôle emploi, etc.). Le déplacement révèle ainsi avec insistance les procédés disciplinaires de quadrillage de l'espace et de contrôle des mobilités humaines (Foucault 1975; Castel 1995; Roche 2003). L'intersaison est aussi ce temps où les travailleurs saisonniers apprennent à composer avec l'instabilité des salaires et l'éphémère intensité des relations sociales qui caractérisent la saisonnalité.

\section{$\&$}

Revenons maintenant sur ce qui ressort de cette analyse de l'expérience des « nourrislogés ». J'ai montré que, dans un cadre où la promiscuité s'impose chaque jour, l'investissement de chacun dans les relations de sociabilité avec les collègues de travail reste nécessaire, bien que pesant. Dans un premier temps, cela crée un mouvement centrifuge vers l'espace du centre de vacances. L'isolement a tendance à être collectif durant la saison, alors que le groupe de pairs éclate aux intersaisons ${ }^{18}$. Les saisonniers construisent les usages de leur temps libre comme des exutoires au « module d'enfermement » que représente l'établissement du centre de vacances. Les distinctions qui en résultent se 
révèlent d'autant plus importantes qu'elles mettent en relief les manières dont chacun tente de se détacher du modèle rationnel de contrôle du travail, de la vie collective dans le centre et au-delà, de la condition sociale qu'entraîne ce type d'emploi. « Le nécessaire repli sur la sphère privée » (Pichon 2002 : 12) se maintient par l'invention de diverses tactiques d'évitement qui rendent l'espace habitable, mais « ce repli » est bien souvent mis à mal par des ingérences diverses.

En ouverture de cet article, j'ai signalé diverses injonctions à l'invisibilité (dans la conception des cadres bâtis, dans les logiques d'installation et l'accès au logement). En restituant des données de terrain ancrées dans le quotidien des « nourris-logés », j’ai indiqué que l'invisibilité des saisonniers dans les espaces du centre a valeur de règle à respecter. Une fois le service fourni, ils doivent disparaître... Une certaine dévalorisation sociale des saisonniers est ainsi entretenue à la fois par le système touristique et sa multiplicité d'acteurs, ainsi que par les contraintes et les prescriptions qui orientent les tâches journalières et qui visent précisément à masquer le travail et les travailleurs pour « enchanter »le touriste. Les quelques clients qui apprirent la présence d'employés logés sur place réagirent avec grand étonnement ${ }^{19}$. Dissimuler les travailleurs saisonniers qui vivent pourtant à l'intérieur même du centre de vacances, voilà un objectif qui paraissait perdu d'avance, mais l'organisation du travail parvient pourtant à ses fins. Le résultat s'impose comme une double fragilisation sociale, à la fois par le régime d'embauche et par les cadres du quotidien (habitat, place dans l'entreprise, sociabilités) qui œuvrent ensemble et empêchent les projections de soi dans l'avenir. Un avenir où le « chez-soi » se dérobe face au travail, contrainte économique urgente, renouvelée chaque fois que la saison touristique approche.

Avec l'ethnographie, l'habitation discrète des «nourris-logés » se donne à voir. C'est un espace non-autonome, non-familial, non-transmissible, temporaire. Un intérêt porté à l'étude de l'habitat (première nécessité des mobiles pour travailler) est une source précieuse pour penser ce mécanisme d'invisibilisation sociale, dont les saisonniers « nourris-logés ", pris dans une dynamique d'insertion (sur un territoire et dans le marché du travail), font une expérience exacerbée. Il reste qu'aucun des saisonniers rencontrés lors de cette enquête ne retourna vivre au centre, même si trois d'entre eux (sur sept) revinrent y travailler. Cette forme d'habitat s'avère être transitoire. Le mode « nourri-logé », en niant une autonomie suffisante des sujets dans leurs espaces intimes, demeure particulièrement infantilisant. Ce type d'habitation semble rejeter hors du territoire ceux qui ne s'y font pas, tout en encourageant d'autres à créer leurs propres réseaux pour trouver les moyens de l'éviter, s'il en est. 


\section{NOTES}

Image d'ouverture:

Àl'occasion des campagnes estivales de la CFDT, le dessinateur Philippe Vuillemin a produit divers dessins sur « le monde du travail saisonnier » (agriculture et tourisme) dont la vocation était de souligner des aspects problématiques de cette forme d'emploi. Les scénettes caricaturales mettent à jour des rapports de subordination habituellement invisibles pour les vacanciers. On voit ainsi dans l'image d'ouverture que la période travaillée (durant les vacances) et le rythme de l'emploi (saisonnier) semblent influer sur les conditions de travail (difficulté d'obtenir un repos). Le commentaire de l'employeur, mettant en parallèle deux termes synonymes (un repos durant les vacances!), provoque l'effet humoristique tout en révélant un état de fait somme toute banal, mais qu'une certaine « mythologie » (Barthes 1957) des vacances participe à dissimuler: des individus travaillent pour produire les vacances des autres.

1. Ce chiffre représente une estimation déjà ancienne pour l'échelle nationale (Le Pors 1999) mais les données statistiques sur cette population sont aujourd'hui essentiellement locales. Chacune des enquêtes chiffrées insiste sur la difficulté d'obtenir des données fiables sur les saisonniers, notamment en raison de la diversité des statuts professionnels liés à la pluri-activité, de la forte mobilité des travailleurs et de l'impossibilité, dans les sources disponibles, de distinguer les CDD de remplacement des CDD saisonniers.

2. Notons toutefois les ouvrages de Nicole Mathieu (1995), ainsi que ceux de Brigitte Biche \& al. (1995).

3. Il faut attendre 1999 pour qu'un rapport ministériel sur les conditions de vie des saisonniers du tourisme (Le Pors 1999) pointe l'épineuse question du logement. Depuis cette date, bien que la plupart des propositions inscrites dans l'expertise aient été sans suites, les collectivités territoriales et le milieu associatif local se sont davantage saisis de cette problématique.

4. Lauteure précise également que la relation salariale de type paternaliste s'avère inégalitaire à plus d'un titre. Par exemple, elle profite davantage aux employés locaux ou originaires des villages proches.

5. Rapport d'étude INSEE PACA (2007 : 1)

6. Hormis dans les entreprises de remontées mécaniques et dans certaines entreprises de tourisme social. Cette mesure vise en partie à éviter la requalification du CDD saisonnier en CDI.

7. C'était le cas au moment où ont été menées les enquêtes de terrain (2006-2011). Cette mesure a pris fin en juillet 2011, elle sera renégociée en 2014 dans le cadre de la convention UNEDIC.

8. Ces difficultés d'installation pour les camions sont également dues aux réglementations pour l'homologation des véhicules.

9. Ces remarques émergent d'une enquête de terrain
(2006-2011) dans le cadre d'une thèse de doctorat en cours. L'enquête a été menée au sein d'une association locale qui engageait des actions en faveur des travailleurs saisonniers, ainsi que dans divers établissements (centres de vacances, remontées mécaniques), en optant pour l'occupation de différents postes de travail. Ces diverses phases de terrain ont été accompagnées du suivi d'une vingtaine de saisonniers durant ces quatre années d'enquête.

10. Qui renvoie notamment à une catégorie usitée dans le traitement des données statistiques.

11. Comme le précise Christian Bromberger (in Bonte \& Izard 1991 : 317): « Micro-lieu construit ou aménagé pour la résidence des hommes, (...) l'habitation apparaît, en fait, comme la résultante d'une pluralité de facteurs (pédologique, climatiques, historiques, économiques, sociaux, symboliques, etc.) que chaque société ordonne et hiérarchise selon des modalités qui lui sont propres. (...) Le but d'une étude ethnologique de la maison est de mettre au jour ce jeu complexe de critères, d'exigences, de limites qui interfèrent dans la production et l'occupation d'un espace bâti ».

12. Terme couramment utilisé par les enquêtés.

13. Pour caractériser ces situations Erving Goffman parle « d'inceste institutionnel » à propos des institutions totalitaires: « Il arrive aussi, comme s'il s'agissait d'un inceste institutionnel, que ces relations se heurtent à une sorte de tabou qui a pour fonction d'empêcher les couples de créer leur propre univers dans l'institution. » (Goffman 1968: 104).

14. Cette anomalie du système de ventilation atteint principalement les deux premiers étages et plus particulièrement les chambres assignées aux saisonniers.

15. Pratique qui consiste à utiliser un temps au travail pour réaliser des activités interdites ou non prévues dans l'emploi du temps. 
16. J'emploie cette expression à la manière de Pascale Trompette dans son enquête sur une usine nucléaire: « le quant à soi incarne une indépendance conquise par rapport aux chaînes réelles et imaginaires du travail » (Trompette 2002: 60).

17. Les saisonniers nourris-logés rencontré lors de cette enquête sont originaires de la côte Varoise et de la côte Atlantique. L'une d'entre eux est originaire d'Alsace. Ils ont tous à nouveau occupé un emploi saisonnier dans la restauration l'été suivant.
18. Maurice Duval (1998) relève le même type de dynamique sociale dans son enquête sur les marins au long cours.

19. Les clients présents lors d'un échange à ce propos ne pouvaient pas croire que «ce détail » avait pu leur échapper. Pour certains d'entre eux, clients « habitués », c'était l'occasion de s'apercevoir qu'ils ne savaient rien des employés qu'ils côtoyaient pourtant quotidiennement depuis deux semaines.

\section{RÉFERENCES}

Baldacchino, G. 1997 Global tourism and informal labour relations. The small-scale syndrome at work. London: Mansell.

Bianchi, R.-V. 2000 Migrant Tourist-Workers: Exploring the "Contact Zones" of Post-industrial Tourism, Current Issues of tourism 3 (2): 107-137.

Biche, B., Gerbaux, F., Le Monnier J. \& al. 1996 Créer son emploi en milieu rural. Les chemins de traverse de la pluriactivité. Paris: L'Harmattan.

Bonnin, P. 1994 L'Habitation, modalité de l'existence sociale. In M. Augé, M. Bassand \& R. Beaunez (dir.), Les Hommes, leurs espaces et leurs aspirations. Hommage à Paul-Henry Chombart de Lauwe. Paris: L'Harmattan.

Bromberger, C. 1991 Habitation, in P. Bonte \& M. Izard, Dictionnaire de l'ethnologie et de l'anthropologie. Paris: Presses Universitaires de France: 317-320.

Castel, R. 1995 Les Métamorphoses de la question sociale: une chronique du salariat. Paris: Gallimard.

De Certeau, M. 1990[1980] L'Invention du quotidien, I. Arts de faire. Paris: Gallimard.

De Certeau, M., Giard, L., Mayol, P. 1994 [1980] L'invention du quotidien, II. Habiter, cuisiner. Paris: Gallimard.

Dehoorne, O., 2002 Tourisme, travail, migration: interrelations et logiques mobilitaires, Revue Européenne des Migrations Internationales 18 (1): 7-36.

Dethyre, R. 2007 Avec les saisonniers. Une expérience de transformation du travail dans le tourisme social. Paris: La Dispute.

Dumazedier, J. 1962 Vers une civilisation du loisir? Paris : Seuil.

Duval, M. 1998 Ni morts, ni vivants: marins! Pour une ethnologie du huis clos. Paris: Presses Universitaires de France.

Flamant, N. \& Jeudy- Ballini, M. 2002 Le Charme discret des entreprises, Terrain 39: 5-16.

Foucault, M. 1975 Surveiller et punir. Naissance de la prison. Paris: Gallimard. 
Friedmann, G. 1956 Le Travail en miettes. Paris: Gallimard.

Giraud, C. 2007 Recevoir le touriste en ami. La mise en scène de l'accueil marchand en chambres d'hôtes, Actes de la Recherche en Sciences Sociales $170: 14-31$.

Gmelch, G. 2003 Behind the smile: the working lives of Caribbean tourism. Bloomington: Indiana University Press.

Goffman, E. 1968 Asiles, études sur la condition sociale des malades mentaux. Paris: Éditions de Minuit.

Guigo, D. 1991 Les Termes d'adresse dans un bureau parisien, L'Homme XXXI (3) : 41-59.

Hughes, E. 1996 Le Regard sociologique. Paris: Éditions de l'EHESS.

Jeanjean, A. 2011 Des-Équilibres humains. Une introduction à «Habiter le temporaire », Techniques \& Culture 56: 16-29.

Kergoat, J., Boutet, J., Jacot, H., Linhart, D., (dir.) 1998 Le Monde du travail. Paris: La Découverte.

Lamanthe, A., 2008 Les Paradoxes de la formalisation salariale en milieu rural (Briançonnais), Études Rurales 182 (2): 29-44.

Le Pors, A. 1999 Propositions pour l'amélioration de la situation sociale et professionnelle des travailleurs saisonniers du tourisme. Paris: La Documentation Française.

Leroi-Gourhan, A. 1964 Le Geste et la parole, Tome II: La Mémoire et les rythmes. Paris: Albin Michel.

Mathieu N. 1995 L'Emploi rural, une vitalité cachée. Paris: L'Harmattan.

Mauss, M. 2006 [1950] Essai sur les variations saisonnières de sociétés Esquimaux. Étude de morphologie sociale. In Sociologie et Anthropologie. Paris: Presses Universitaires de France. : 389-476.

Michaud J., 2001 Anthropologie, tourisme et sociétés locales au fil des textes, Anthropologie et sociétés 25 (2): 15-33.

Monjaret, A. 2001 La Fête: une pratique extraprofessionnelle sur les lieux du travail, Cités 8: 87-100.

Pétonnet, C. 2002 On est tous dans le brouillard. Paris: Éditions du CTHS.

Pichon, P. 2002 Vivre sans domicile fixe: l'épreuve de l'habitat précaire, Communications 73: 11-29.

Réau, B. 2006 Les Devoirs de vacances: la vie quotidienne d'un Gentil Organisateur du Club Méditerranée, Regards Sociologiques $32: 73-81$.

Roche, D. 2003 Humeurs vagabondes: de la circulation des hommes et de l'utilité des voyages. Paris: Fayard.

Rosselin, C. 2002 L'Observation des pratiques habitantes dans les logements d'une seule pièce, Communications 73: 95-112.

Sénépart, I. 2011 Nomades ou sédentaires mobiles? Ancrage archéologique et historique de l'habitat temporaire, Techniques \& Culture 56: 30-47.

SUD INSEE, 2007 Les Saisonniers d'hiver de la montagne en Provence Alpes Côte d'Azur, Rapport d'étude 107.

[En ligne], consulté le 06 juin 2012, http://www.adrets-asso.fr/IMG/pdf/salaries_saisonnier_hiver_2004_ INSEE.

Thalineau, A. 2002 L’Hébergement social: espaces violés, secrets gardés, Ethnologie française XXXVII : 4148.

Trompette, P. 2002 Un Rayon de soleil dans l'atelier... Le quotidien du travail dans usine nucléaire, Terrain 39: 49-68.

Uriely, N. 2001 "Travelling workers" and "working tourists" : variations across the interaction between work and tourism, International Journal of Tourism Research 1 (3): 1-8.

Veblen, T. 1970 [1899] Théorie de la classe de loisir. Paris: Gallimard.

Viard, J. 2002 Le sacre du temps libre: la société des 35 heures. La tour d'Aigues: Éditions de L'Aube.

Weber, F. 1986 Le Travail à côtée étude d'ethnographie ouvrière. Paris: INRA/EHESS.

Winkin Y. 2001 Propositions pour une anthropologie de l'enchantement. In P. Rasse, N. Midol \& F. Triki (dir) Unité-diversité. Les identités culturelles dans le jeu de la mondialisation. Paris: L'Harmattan: 169-179.

Wozniak, M. 2002 Les Stations de ski: quelles représentations des clientèles pour quel cadre bâti?, Revue de géographie alpine, T. 90, n 4: 17-31. 


\section{RÉSUMÉ}

Lorsque le « chez-soi » se dérobe: invisibilisation des travailleurs saisonniers en montagne, le cas des "nourris-logés". Les activités de travail en centre de vacances donnent naissance à un type spécifique d'habitat: "nourri-logé ». Lauteure se propose, dans cet article, de décrire et de formaliser une analyse de ce mode d'habitat temporaire qu'est celui des « nourris-logés » en saison. Elle s'attache à montrer comment l'imbrication du lieu de vie à celui du travail, agit sur la façon dont les saisonniers habitent l'espace-temps du centre de vacances. L'évocation des interférences entre sphère privée et publique, de la promiscuité et du contrôle social exacerbé que ce mélange produit, la conduit également à aborder des mécanismes de distinction qui prennent leur source dans ce que chacun décide de faire de son temps libre. Larticle renvoie de ce fait à la question essentielle de la définition de frontières entre sphère privée et travail, sans omettre une réflexion portant sur un temps autre, l'intersaison, temps du retour et de la confrontation à l'à-venir. L'approche ethnographique de l'habitat des «nourris-logés » s'avère être une source précieuse pour penser la condition sociale des saisonniers du tourisme, dont l'auteure montre qu'elle est marquée par un mécanisme d'invisibilisation.

\section{ABSTRACT}

When " at home " slips away. Invisibilisation of the seasonal workers in mountain: the case of "board and lodging ". Holiday work activities give rise to a specific type of housing environment : « board and lodging ». In this article, the author suggests to describe and formalize an analysis of this mode of temporary housing environment. She seeks to show how the nesting place of life in the one of the work, acts on the way the seasonal workers live in the space-time of the vacation center. Evocation of interference between private and public sphere, of promiscuity and exacerbated social control that this product mix, also leads her to address distinction mechanisms taking their source in what everyone decides to do of their free time. The article returns to the critical issue of the definition of boundaries between private life and work, without omitting a reflection on another time, the inter-season, time for the return and the confrontation with the future. The ethnographical approach of the «board and lodging " environment turns out to be a precious source to think of the social condition of the seasonal workers of the tourism, whose author shows that it is marked by a mechanism of invisibilisation.

\section{MOTS-CLÉS}

Saison touristique, « nourris-logés », habitat, temporalités, appropriation, travail/hors travail, promiscuité, sociabilité.

\section{KEYWORDS}

Tourist season, « board and lodging », housing environment, temporality, appropriation, work/ except work, promiscuity, sociability. 